\title{
The chemical chaperon 4-phenylbutyric acid ameliorates hepatic steatosis through inhibition of de novo lipogenesis in high-fructose-fed rats
}

\author{
LU-PING REN ${ }^{1}$, GUANG-YAO SONG ${ }^{1}$, ZHI-JUAN HU ${ }^{2}$, MINGMING ZHANG ${ }^{3}$,

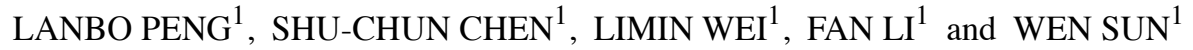 \\ Departments of ${ }^{1}$ Endocrinology and ${ }^{2}$ Nephrology, ${ }^{3}$ Clinical Laboratory, \\ General Hospital of Hebei, Shijiazhuang, Hebei 050051, P.R. China
}

Received May 16, 2013; Accepted August 19, 2013

DOI: 10.3892/ijmm.2013.1493

\begin{abstract}
Non-alcoholic fatty liver disease caused by dietary factors such as a high fructose intake is a growing global concern. The aim of this study was to investigate the intervention effects of an endoplasmic reticulum stress (ERS) inhibitor 4-phenylbutyric acid (PBA) on liver steatosis induced by high-fructose feeding in rats and the possible underlying mechanisms. Wistar rats were divided into the control, high-fructose group (HFru) and PBA intervention (HFru-PBA) groups. PBA intervention was initiated following 4 weeks of high-fructose feeding. After 8 weeks of feeding, the ERS markers p-PERK, p-eIF2 $\alpha$, p-IRE-1, spliced XBP-1, ATF-6 were measured by western blotting. Liver triglyceride contents and morphological changes were examined. The protein expression of lipogenic key enzymes (ACC, FAS and SCD-1) and upstream transcriptional factors (SREBP-1c and ChREBP) were measured. The ERS-related cell events, oxidative stress and apoptosis, were evaluated by standard methods. Results demonstrated that PBA intervention significantly resolved hepatic ERS and improved liver steatosis induced by high-fructose feeding in rats. The protein expression of ACC, FAS, SCD-1 and SREBP-1c was upregulated in high-fructose-fed rats, whereas it decreased following PBA intervention. Oxidative stress and apoptosis were observed in livers of high-fructose-fed rats, but were alleviated by PBA intervention. ERS is involved in the development of fatty liver induced by a high fructose intake. ERS inhibition by PBA can therefore ameliorate liver steatosis through inhibition of hepatic lipogenesis.
\end{abstract}

Correspondence to: Professor Guang-Yao Song, Department of Endocrinology, General Hospital of Hebei, Heping Xi Road 348, Shijiazhuang, Hebei 050051, P.R. China

E-mail: sguangyao2@163.com

Key words: endoplasmic reticulum stress, fatty liver, fructose, lipogenesis

\section{Introduction}

Non-alcoholic fatty liver disease (NAFLD) has gained increasing attention worldwide due to its prevalence and its association with insulin resistance and metabolic syndrome $(1,2)$. Hepatic steatosis is the basic pathophysiological change occurring throughout the development of NAFLD. Dietary effects on whole-body metabolism and its regulation via the effects on lipid metabolic pathways are considered to be crucial in the pathogenesis of hepatic steatosis $(3,4)$. The effect of high fructose intake on the pathogenesis of hepatic steatosis due to an increase in daily fructose intake and its harmful impact on hepatic lipid metabolism has gained much attention (5-7). High-fructose feeding leads to significant lipid accumulation in livers of rodents (7-9). One important reason for this is that dietary fructose stimulates endogenous de novo lipogenesis within the liver (10). However, the clear underlying mechanisms by which fructose induces liver steatosis remain to be clarified.

Previous studies demonstrated that endoplasmic reticulum stress (ERS) and unfolded protein response (UPR), which occurs following ERS, have a regulatory effect on lipid synthesis in liver (11-13). On the other hand, ERS is associated with the development of NAFLD. Specificallly, hepatic ERS is accompanied by a fatty liver in genetically obese or chronic high-fat-fed rodents $(14,15)$. An intervention study on ERS inhibitors, 4-phenylbutyric acid (4-PBA) and tauroursodeoxycholic (TUDCA) found that resolved ERS is able to ameliorate hepatic steatosis in ob/ob mice $(15,16)$. In a short-term study, it was suggested that ERS is involved in the development of lipid accumulation in liver in mice fed a high fructose diet (17). Few studies have investigated the role of ERS in hepatic steatosis induced by long-term high-fructose feeding.

The aim of the present study was to clarify the role of ERS in the development of fatty liver induced by long-term high fructose intake. Fructose is a dietary factor that highly stimulates lipogenesis, while ERS has a regulatory effect on lipogenesis. We hypothesized that alleviation of ERS is able to ameliorate hepatic steatosis in high-fructose-fed rats through regulation of de novo lipogenesis. To prove this hypothesis, 4-phenylbutyric acid was used to inhibit the ERS induced by 
high-fructose feeding in liver in Wistar rats. Lipid content, ERS and lipogenic markers in liver were detected in order to investigate the association between ERS and fructose-induced fatty liver. Oxidative stress and hepatocyte apoptosis were also observed to study the role of ERS in the development of NAFLD.

\section{Materials and methods}

Animals. Male Wistar rats supplied from the Experimental Animal Center of Hebei Province (Shijiazhuang, China) were conditioned in communal cages for 1 week at $22.0 \pm 0.5^{\circ} \mathrm{C}$ with a 12/12-h light/dark cycle (lights on 06.00 a.m.). Experimental procedures were approved by the Animal Ethics Board of the Hebei Research Institute for Endocrine and Metabolic Diseases and were in accordance with China's National Code of the Animal Care for Scientific Experimentation. After an acclimatization period, the rats (250-300 g) were divided into three groups. The control group (Con) was fed a standard laboratory diet (18). The high-fructose (HFru) and 4-phenylbutyric acid (PBA) intervention (HFru-PBA) groups were fed a highfructose diet [35\% calories from fructose, $35 \%$ calories from starch, $\sim 9 \%$ calories from fat and $21 \%$ calories from protein; based on a recipe described in a study by Ren et al (17)]. PBA [dose: $0.35 \mathrm{~g} / \mathrm{kg}$.day, based on a previous study (15)] was administered to the HFru-PBA group by oral gavage subsequent to 4 weeks of high-fructose feeding. After 8 -weeks, the rats were sacrificed and liver tissues were collected.

Plasma glucose concentrations were determined using a glucometer (Accu-Check Active, Roche Diagnostics GmbH, Mannheim, Germany). Plasma insulin was measured using a radioimmunoassay kit (Linco Research, St. Charles, MO, USA). The enzymatic activities of ALT and AST were determined using a Biochemical Analyzer (Beckman X20, Beckman Coulter, Brea, CA, USA). Plasma FFA concentration was measured using an enzymatic colorimetric method (NEFA $\mathrm{C}$ test kit, Jiancheng Biological Corporation, Jiangsu, China). Plasma triglyceride concentrations and liver triglycerides (extracted from homogenated liver tissues) were determined by a Peridochrom Triglyceride GPO-PAP kit (Boehringer Mannheim, Mannheim, Germany).

Histology staining. Small liver sections, fixed in $10 \%$ buffered formalin, were processed for embedding in paraffin. Sections of 5-6 $\mathrm{mm}$ were cut for histopathological evaluation. Liver sections were stained with hematoxylin and eosin (H\&E staining) using a standard protocol and then analyzed by light microscopy.

Hyperinsulinemic-euglycaemic clamp. The study was conducted between 09.00 and 12.00 a.m. at week 8 in animals that had been fasted for $12 \mathrm{~h}$. After being anaesthetized with pentobarbitone $(40 \mathrm{mg} / \mathrm{kg}$, i.p.), the rat was placed on a warm table to maintain rectal temperature at $37^{\circ} \mathrm{C}$. Catheters were inserted into the left femoral vein (for infusion of glucose and insulin) and the femoral artery (for blood sampling). After a basal period of $30 \mathrm{~min}$, a hyperinsulinaemic-euglycaemic clamp was performed, as previously described (19). In brief, human insulin (Actrapid; Novo-Nordisk, Beijing, China) was infused at a constant rate of $4.1 \mathrm{mU} / \mathrm{kg}$ per min to achieve physiological hyperinsulinaemia $(100-150 \mathrm{mU} / \mathrm{l})$ for a period of $2 \mathrm{~h}$. The blood glucose concentration was clamped at the basal level by infusing glucose at variable rates. Under these conditions, the glucose infusion rate (GIR) required to maintain euglycaemia (usually calculated between 60 and $120 \mathrm{~min}$ ) reflects whole-body insulin sensitivity.

Western blot analysis. Liver samples were homogenized in ice-cold lysis buffer $(50 \mathrm{mM}$ Tris $\mathrm{pH} 7.5,150 \mathrm{mM} \mathrm{NaCl}$, $1 \%$ Triton X-100, $10 \mathrm{mM} \mathrm{NaP}, 100 \mathrm{mM} \mathrm{NaF}, 2 \mathrm{mM} \mathrm{Na}$ orthovanadate, $1 \mathrm{mM}$ EDTA, $1 \mathrm{mM}$ EGTA, $10 \%$ glycerol), supplemented with protease inhibitor cocktail tablets (Roche) and DL-dithiothreitol, and solubilized for $30 \mathrm{~min}$ at $4^{\circ} \mathrm{C}$. Protein samples were then denatured in SDS sample buffer (125 mmol/1 Tris-HCl, pH 6.8, 50\% glycerol, 2\% SDS, $5 \% \beta$-mercaptoethanol and $0.01 \%$ bromophenol blue). Equal amounts of tissue lysates were resolved by SDS-PAGE and immunoblotted with antibodies against the following proteins: ERS markers, phosphorylated pancreatic ER kinase (p-PERK) (Thr980, Cell Signaling Technology, Danvers, MA, USA), total- and phospho (Ser51)-eukaryotic translation initiation factor $2 \alpha$ (eIF2 $\alpha$, Santa Cruz Biotechnology, Santa Cruz, CA, USA), inositol-requiring kinase 1 (IRE1, Abcam, Cambridge, MA, USA), phospho-IRE1 (Ser724, Abcam), X-box banding protein-1 (XBP1, Santa Cruz Biotechnology), activation transcriptional factor 6 (ATF6, Santa Cruz Biotechnology), C/EBP homologous protein (CHOP, Cell Signaling Technology); upstream transcriptional factors of lipogenesis: sterol regulatory element-binding protein1c (SREBP1c, Santa Cruz Biotechnology), carbohydrate responsive element binding protein (ChREBP, Santa Cruz Biotechnology); downstream lipogenic enzymes, acetyl-CoA carboxylase (ACC, Upstate, Lake Placid, NY, USA), fatty acid synthase (FAS, Abcam) and stearoyl-CoA desaturase 1 (SCD1, Cell Signaling Technology). Antibodies against $\beta$-actin (Santa Cruz Biotechnology) were blotted in each gel as the loading control.

Determination of oxidative parameters. Tissue homogenates were centrifuged for $15 \mathrm{~min}$ at $15,000 \mathrm{x} \mathrm{g}$, and then the clear supernatants were removed for analysis. Lipid peroxidation in the liver was measured by the formation of malondialdehyde (MDA). The levels of MDA and the activities of antioxidant enzymes including SOD, GSH-Px and CAT were assayed using commercial assay kits according to the manufacturer's instructions. The MDA level was expressed as $\mathrm{nmol} / \mathrm{mg}$ protein. The activities of antioxidant enzymes were expressed as $\mathrm{U} / \mathrm{mg}$ protein.

Terminal deoxynucleotidyl transferase-mediated dUTP nick end-labeling method (TUNEL) assay. Paraffin sections (6- $\mu \mathrm{m})$ were collected on poly-L-lysine-coated glass slides, and the nuclear DNA fragmentation of apoptotic cells was labeled in situ with the ApopTag Peroxidase in situ Apoptosis Detection kit (Intergen Co., Purchase, NY, USA). DNA fragmentation was determined using a TUNEL assay as described by Boncompagni et al (20). TUNEL-positive nuclei were counted.

Statistical analyses. The results are presented as means \pm SE. Differences were considered significant when $\mathrm{p}<0.05$ tested 
Table I. Basal characteristics and plasma parameters in three groups of rats at the end of the 8th week (mean $\pm \mathrm{SD}, \mathrm{n}=12$ ).

\begin{tabular}{lccc}
\hline Characteristics & Con & HFru & HFru-PBA \\
\hline Body mass (g) & $385 \pm 20$ & $383 \pm 23$ & $374 \pm 20$ \\
Visceral WAT (g) & $2.5 \pm 0.3$ & $3.7 \pm 0.4^{\mathrm{b}}$ & $3.1 \pm 0.5^{\mathrm{a}, \mathrm{d}}$ \\
TC (mmol/l) & $1.22 \pm 0.10$ & $1.57 \pm 0.14^{\mathrm{b}}$ & $1.37 \pm 0.17^{\mathrm{a}, \mathrm{d}}$ \\
TG (mmol/l) & $0.91 \pm 0.09$ & $1.87 \pm 0.19^{\mathrm{b}}$ & $1.34 \pm 0.16^{\mathrm{b}, \mathrm{d}}$ \\
FFAs (mmol/l) & $0.53 \pm 0.15$ & $1.09 \pm 0.19^{\mathrm{b}}$ & $0.69 \pm 0.11^{\mathrm{a}, \mathrm{d}}$ \\
ALT (IUl/l) & $31 \pm 2$ & $33 \pm 2$ & $31 \pm 3$ \\
AST (IUl/l) & $86 \pm 10$ & $91 \pm 12$ & $82 \pm 10$ \\
FBG (mmol/l) & $4.53 \pm 0.20$ & $5.73 \pm 0.39^{\mathrm{b}}$ & $5.07 \pm 0.29^{\mathrm{b}, \mathrm{d}}$ \\
FINS (ng/ml) & $2.77 \pm 0.48$ & $3.99 \pm 0.80^{\mathrm{b}}$ & $3.39 \pm 0.40^{\mathrm{c}, \mathrm{d}}$
\end{tabular}

Compared with CON group, ${ }^{\mathrm{a}} \mathrm{P}<0.05,{ }^{\mathrm{b}} \mathrm{P}<0.01$. compared with $\mathrm{HFru}$ group, ${ }^{\mathrm{C}} \mathrm{P}<0.05,{ }^{\mathrm{d}} \mathrm{P}<0.01$. WAT, white adipose tissue.

by one-way analysis of variance (ANOVA). When significant variations were found, the Tukey-Kramer multiple comparisons test was applied.

\section{Results}

Baseline characteristics and plasma parameters in three groups of rats fed for 8 weeks. After 8 weeks of feeding, no difference was observed in body mass for the three groups of rats. Visceral white adipose tissue (WAT) was increased in the HFru group compared with the Con group $(\mathrm{p}<0.05)$, while visceral WAT was significantly decreased in the HFru-PBA group compared with the HFru group. Total cholesterol (21), triglyceride (TG) and free fatty acids in plasma were all increased in the HFru group compared with the Con group, but improved in the HFru-PBA group. No difference was observed in plasma ALT and AST for the three groups. Basal plasma glucose and insulin concentrations were both increased in the HFru group compared with the Con group, but decreased in the HFru-PBA group compared with the HFru group (Table I).

Systemic insulin resistance was induced by high-fructose feeding but ameliorated by PBA intervention after 8 weeks of feeding. After 8 weeks of feeding, the GIR during the hyperinsulinemic-euglycaemic clamp was reduced by $48 \%$ in the HFru group compared with the Con group $(\mathrm{p}<0.01)(n=6$ in each group). In the HFru-PBA group, GIR was increased by $46 \%$ through PBA intervention $(\mathrm{p}<0.01)$ (Fig. 1).

PBA intervention ameliorated high-fructose feedinginduced hepatic steatosis after 8 weeks. After rats were fed for 8 weeks, the liver triglyceride content was increased by 1.8 -fold $(\mathrm{p}<0.01)$ in the HFru group compared with the Con group. PBA intervention decreased liver triglyceride content by $43 \%$ ( $\mathrm{p}<0.01)$ (Fig. 2A). H\&E staining revealed marked vacuolar degeneration in the liver in the HFru group at the end of the 8th week, indicating fat accumulation in the liver. Reduced liver lipid deposition was observed in the HFru-PBA group (Fig. 2B).

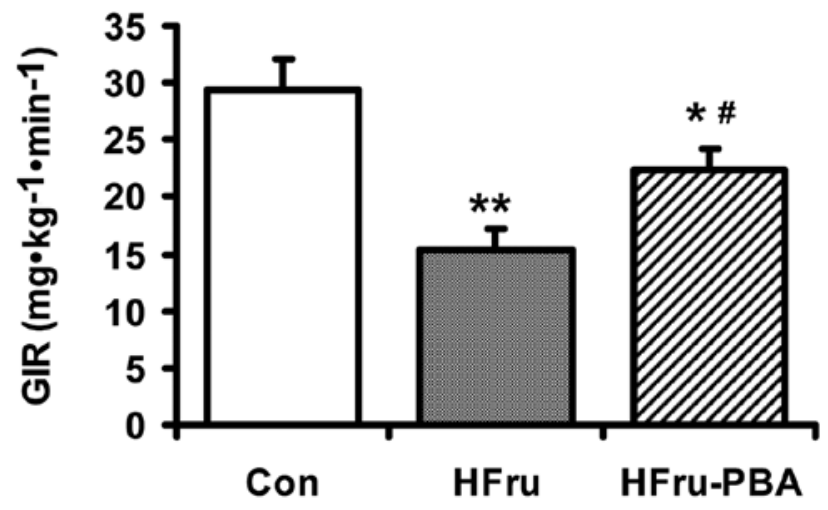

Figure 1. Systemic insulin resistance was induced by high-fructose feeding but ameliorated by PBA intervention after 8 weeks of feeding. Glucose infusion rates in rats in the Con, HFru and HFru-PBA groups during the hyperinsulinemic-euglycaemic clamp study. Data are presented as means \pm SE of 6 rats ${ }^{*} \mathrm{P}<0.05$ vs. Con rats; ${ }^{* *} \mathrm{P}<0.01$ vs. Con rats; ${ }^{\prime \prime} \mathrm{P}<0.05$ vs. HFru rats.

ERS markers were activated by high-fructose feeding but normalized by PBA intervention after 8 weeks of feeding. After 8 weeks of feeding, the protein expression of the activation forms of ERS markers, including phosphorylated PERK (p-PERK), phosphorylated eIF $2 \alpha$ (p-eIF $2 \alpha$ ), phosphorylated IRE-1 (p-IRE-1) , spliced XBP1 (shown as the ratio of spliced XBP1 to unspliced XBP1; spliced XBP1 is the activated form of XBP1) and ATF6, were all upregulated in rat livers in the HFru group (all p-values $<0.01$ ); while the protein expression of the above markers in liver were significantly inhibited in the HFru-PBA group (all p-values <0.01) (Fig. 3).

Protein expression of lipogenic enzymes were stimulated by high-fructose feeding but were decreased by PBA intervention after 8 weeks. Compared with the Con group, protein levels of the key lipogenic enzymes including ACC, FAS and SCD1 were significantly increased by 2.8, 5.7 and 3.8-fold, respectively, in the liver tissues in rats after 8 weeks of high-fructose feeding (all p-values <0.01). After PBA intervention, the proteins levels of ACC, FAS and SCD1 were almost normalized in rat livers in the HFru-PBA group (all p-values <0.01) (Fig. 4).

Upregulated protein expression of SREBPlc induced by highfructose feeding was decreased by PBA intervention after 8 weeks. Protein contents of SREBP1c in rat livers in the HFru group were upregulated by 1.9 and 4.3 -fold, respectively, but downregulated by 66 and 57\%, respectively, with PBA intervention (both p-values $<0.01$ ). Compared with the Con group, the protein expression of ChREBP was increased by $66 \%$ in the HFru group $(\mathrm{p}<0.01)$ but did not change with PBA intervention (Fig. 5).

Oxidative stress in liver tissue in high-fructose-fed rats was relieved by $P B A$ intervention. The MDA level was significantly increased in the HFru group compared with the Con group after 8 weeks of feeding. By contrast, PBA intervention decreased the MDA level significantly in the HFru-PBA group (both p-values <0.01) (Fig. 6A). Compared with the Con group, the activities of SOD, GSH-px and CAT 

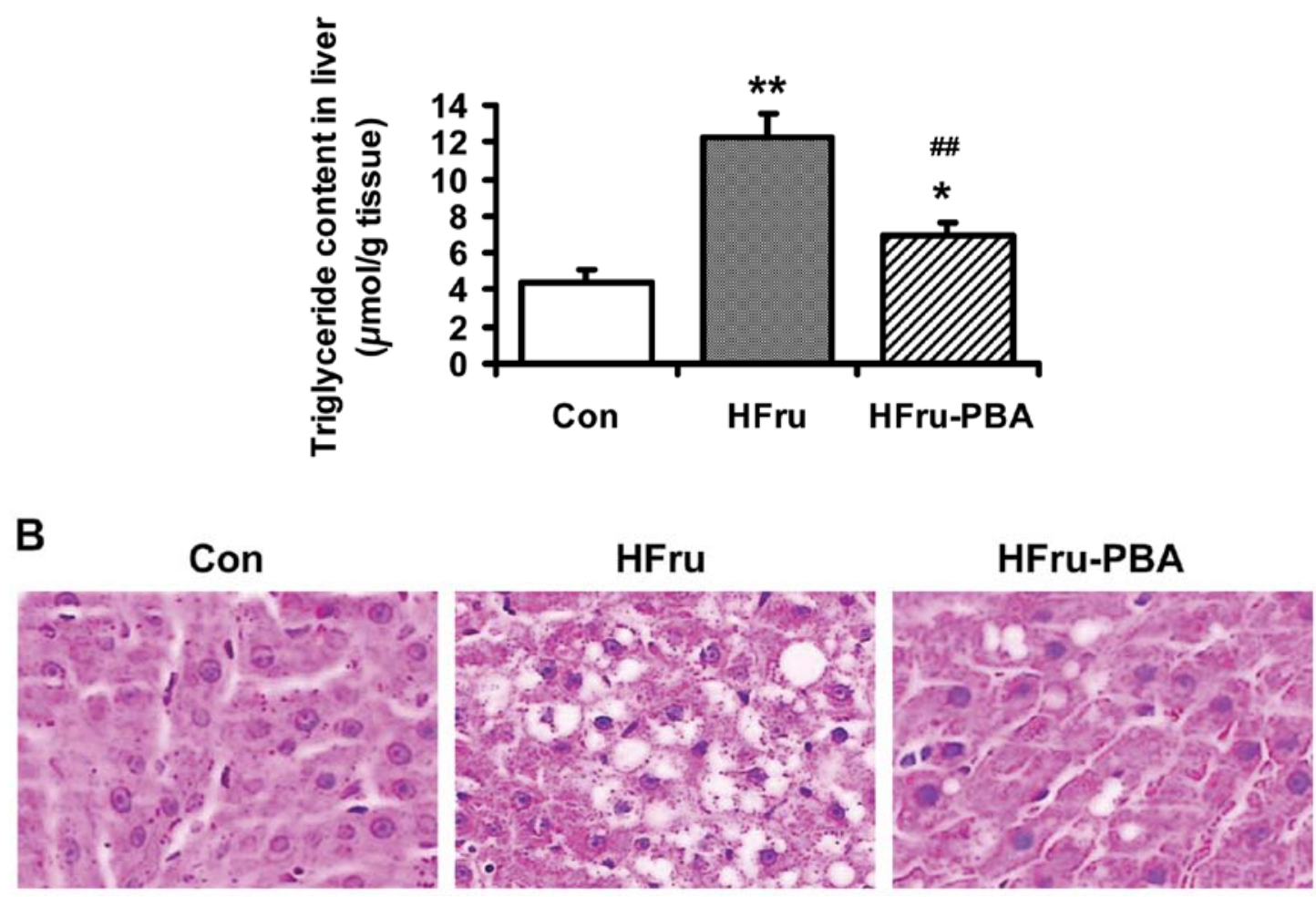

Figure 2. PBA intervention ameliorated high-fructose feeding-induced liver steatosis after 8 weeks of feeding. (A) Triglyceride contents in liver tissues in standard diet-fed (Con), high-fructose-fed (HFru) and 4-phenylbutyric-acid (PBA)-treated (HFru-PBA) rats after 8-weeks of feeding. Data are presented as means \pm SE of $10-11$ rats. ${ }^{*} \mathrm{P}<0.05$ vs. Con rats; ${ }^{* *} \mathrm{P}<0.01$ vs. Con rats; ${ }^{; \#} \mathrm{P}<0.01$ vs. HFru rats. (B) H\&E staining of liver tissues in mice in the Con, HFru and HFru-PBA groups at the end of the 8 th week (H\&E, $x 400, n=6)$.

in in high-fructose-fed rat livers were significantly decreased by $26.4,16.4$ and $23.1 \%$, respectively. In the HFru-PBA group, the activities of the abovementioned parameters in livers were recovered by $18.2,11.1$ and $21.5 \%$, respectively, following PBA intervention (all p-values <0.05) (Fig. 6B-D).

Changes in TUNEL assay and the protein expression of CHOP indicate that apoptosis in hepatocytes was increased in livers in high-fructose-fed rats while it was relieved in livers in high-fructose-fed rats following PBA intervention. Compared with the Con group, the percentage of TUNEL-positive cells in livers was significantly increased $(1.4$ vs. $23.8 \%, \mathrm{p}<0.01)$ in the HFru group. In the HFru-PBA group, PBA treatment decreased the percentage of TUNEL-positive cells in livers to $11.0 \%$ (p<0.01) (Fig. 7A). Compared with the Con group, the protein contents of CHOP in livers were increased by $59 \%$ $(\mathrm{p}<0.01)$ in the HFru group. PBA treatment decreased the protein contents of CHOP by $21 \%$ in rat livers in the HFru-PBA group $(\mathrm{p}<0.05)$ (Fig. 7B).

\section{Discussion}

Animal studies have shown that a high intake of fructose leads to hepatic steatosis and whole-body insulin resistance $(8,9)$. In the present rat study, 8 weeks of high-fructose feeding induced liver lipid accumulation, increased glucose and insulin levels in plasma and decreased GIR during hyperinsulinaemic-euglycaemic clamp study, which are consistent with previous studies (9). The mechanisms by which fructose induces hepatic steatosis remain to be clarified. From previous studies (17), ERS is possibly involved in the development of fatty liver induced by high-fructose feeding.

ERS involves the disruption of endoplasmic reticulum homeostasis. Unfolded protein response (UPR) is the selfprotective mechanism in endoplasmic reticulum required to cope with ERS. Since no direct marker of ERS is currently available, the transcription factors of the three pathways of UPR are used as indirect ERS markers, including the PERK-eIF2 $\alpha$, IRE-1-XBP1 and ATF6 pathways. ERS has been shown to be involved in the development of NAFLD and insulin resistance in genetically obese mice models or high-fat-fed rodent models $(14,15)$. Inhibition of ERS is able to ameliorate hepatic steatosis in ob/ob mice $(15,16)$. Additionally, ERS is induced in mice fed on a short-term high-fructose diet in hepatic steatosis (17). However, few studies have been conducted on the role of ERS in hepatic steatosis induced by long-term intake of fructose. Results of the present study show that the hepatic lipid accumulation induced by long-term high-fructose feeding was accompanied by ERS in liver in Wistar rats, as reflected by the activation of 3' UPR pathways. However, resolved ERS by PBA intervention ameliorated hepatic steatosis, indicating that ERS is involved in the pathogenesis of fatty liver induced by high-fructose feeding. On the other hand, PBA intervention improved the whole-body glucose metabolism and insulin 


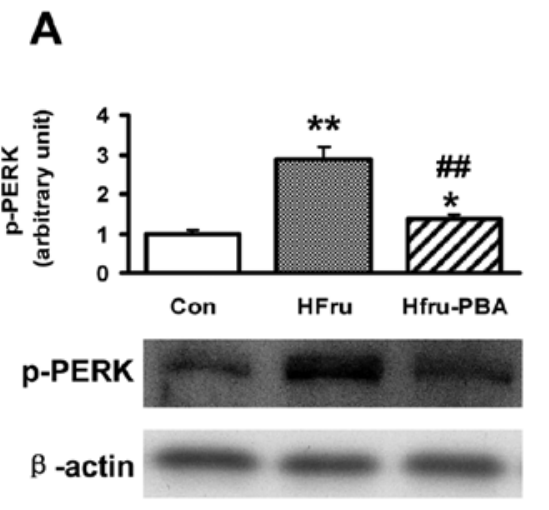

C
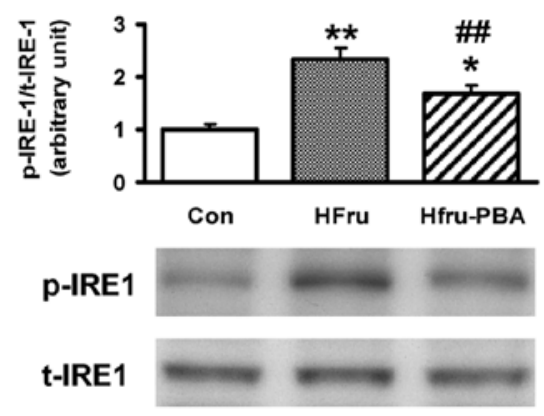

E

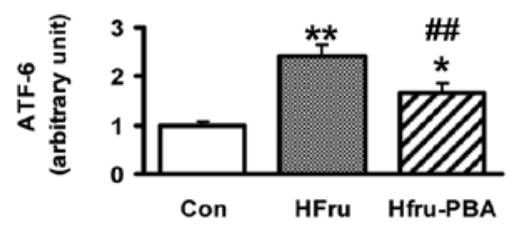

ATF-6

$\beta$-actin
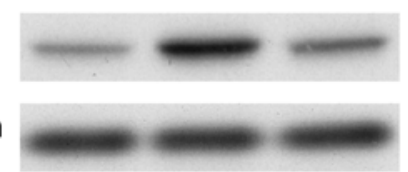

B

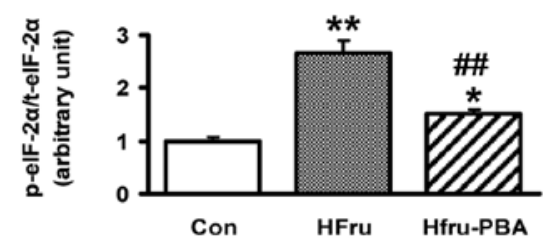

p-elF-2 a

t-elF-2 $a$

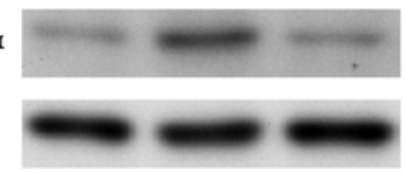

D

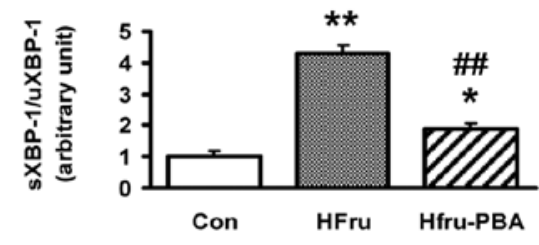

spliced XBP-1 unspliced XBP-1

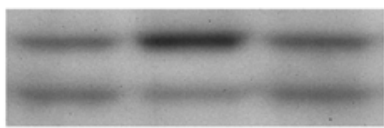

Figure 3. ERS markers were activated by high-fructose feeding but were normalized by PBA intervention after rats were fed for 8 weeks. Protein levels of (A) phosphorylated PERK (p-PERK), (B) phosphorylated eIF2 $\alpha$ (p-eIF2 $\alpha$ ), (C) phosphorylated IRE-1 (p-IRE-1), (D) spliced XBP-1 and (E) ATF-6 in livers in three groups of rats. Data are presented as means $\pm \mathrm{SE}$, and two representative blots of each group were presented for the protein. ${ }^{*} \mathrm{P}<0.05$ vs. Con rats; ${ }^{* *} \mathrm{P}<0.01$ vs. Con rats; ${ }^{*} \mathrm{P}<0.01$ vs. HFru rats $(\mathrm{n}=6)$.

sensitivity as shown by the decreased glucose and insulin levels and increased GIR in the hyperinsulinemic-euglycaemic clamp study. The results confirmed that ERS is associated with insulin resistance.

Fructose is known to be a highly lipogenic dietary factor, and increased hepatic lipogenesis is an important mechanism by which fructose induces hepatic steatosis (22). Studies (11-13) have shown that ERS and UPR have a regulatory effect on the lipid synthesis in the liver. In obese rodents, the ERS-induced dissociation of ERS chaperon GRP78 leads to an upstream lipogenic transcriptional factor SREBP1c maturation and result in hepatic steatosis, indicating ERS has a regulatory effect on SREBP1c (12). On the other hand, the ERS marker along the IRE-1-XBP1 pathway of UPR, XBP1 has been established as a novel transcription factor governing hepatic lipogenesis (13). Since it has been proven that ERS and
UPR have a regulatory effect on the lipid synthesis in liver (11-13), we hypothesized that the alleviation of ERS decrease lipid contents in liver by inhibiting de novo lipogenesis. As shown in the results, the key lipogenic enzymes including ACC, FAS and SCD1 were all upregulated by high-fructose feeding, which reflects increased de novo lipogenesis, while in the Fru-PBA group, the protein expression of ACC, FAS and SCD1 was significantly decreased. This finding confirms our hypothesis and suggests that resolved ERS by PBA is capable of inhibiting de novo lipogenesis and thus ameliorating hepatic steatosis. We then assessed the abovementioned upstream transcriptional factors, including SREBP1c, ChREBP [another well-known upstream transcriptional factor of de novo lipogenesis in liver (23)] and spliced XBP1. The results demonstrated that long-term fructose intake increased the protein expression of SREBP1c, ChREBP and spliced XBP1, whereas PBA 
A

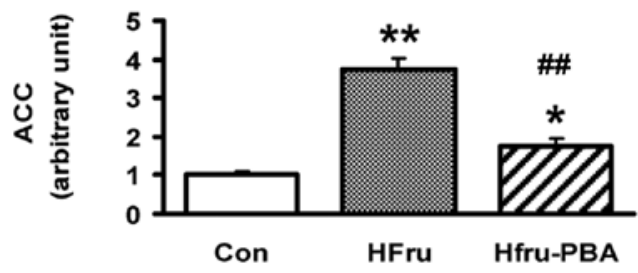

ACC

\section{$\beta$-actin}

B

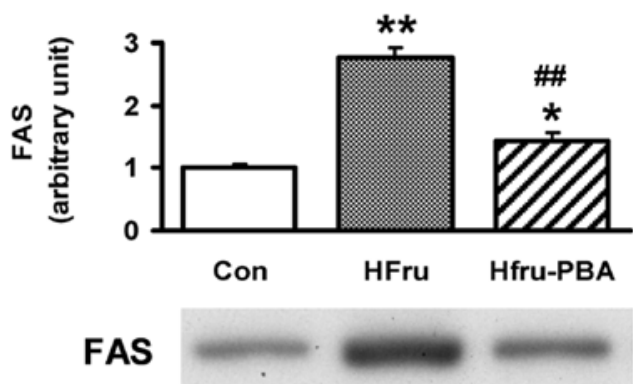

$\beta$-actin

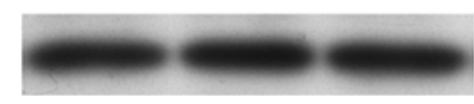

C

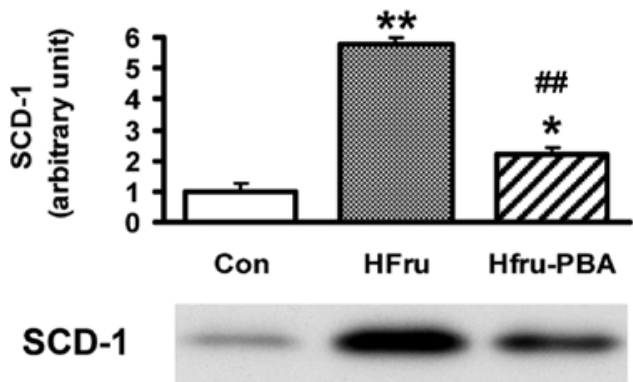

$\beta$-actin

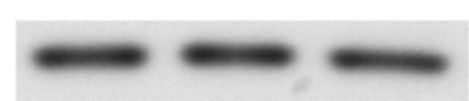

Figure 4. Protein expression of lipogenic enzymes was stimulated by high-fructose feeding but was decreased by PBA intervention after 8 weeks. Protein levels of (A) ACC, (B) FAS and (C) SCD-1 in livers in three groups of rats. Data are presented as means $\pm \mathrm{SE}$, and two representative blots of each group were provided for the protein. ${ }^{*} \mathrm{P}<0.05$ vs. Con rats; ${ }^{* *} \mathrm{P}<0.01$ vs. Con rats; ${ }^{\# \#} \mathrm{P}<0.01$ vs. HFru rats $(\mathrm{n}=6)$.

intervention decreased the protein levels of SREBP1c and spliced XBP1, but not ChREBP. Combined with the observation that ACC, FAS and SCD1 in liver were downregulated following PBA intervention, the results indicate that ERS has a regulatory effect on the lipogenesis. Decreased expression of SREBP1c and XBP1 may contribute to the decreased protein expression of key lipogenic enzymes, resulting in improved
A

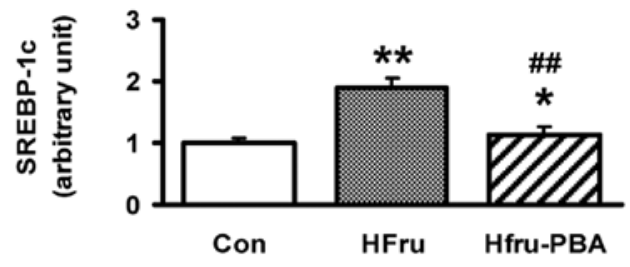

SREBP-1

$\beta$-actin

B

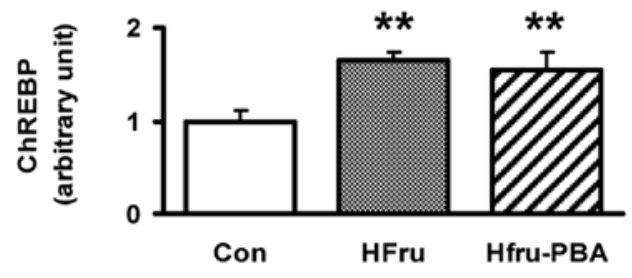

ChREBP

$\beta$-actin
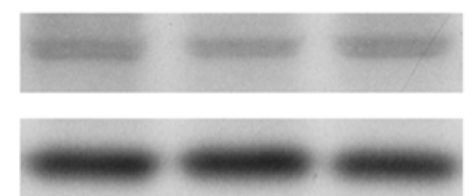

Figure 5. Change in the protein expression of SREBP-1c and ChREBP in three groups of rats after 8 weeks of feeding. Protein levels of (A) SREBP-1c and (B) ChREBP in livers in three groups of rats. Data are presented as means $\pm \mathrm{SE}$, and two representative blots of each group were given for the protein. ${ }^{*} \mathrm{P}<0.05$ vs. Con rats; ${ }^{* *} \mathrm{P}<0.01$ vs. Con rats; ${ }^{\# \#} \mathrm{P}<0.01$ vs. HFru rats $(\mathrm{n}=6)$.

hepatic steatosis. Of note, the protein expression of ChREBP was not changed by PBA intervention. However, the association between ChREBP and ERS remains to be clarified. Our results show that ERS inhibition does not affect the expression of ChREBP, suggesting that ERS does not have a regulatory effect on ChREBP.

Another finding in our study is that ERS and oxidative stress occur simultaneously in livers in high-fructose-fed rats. Inhibition of ERS by PBA alleviated oxidative stress in liver induced by high-fructose feeding, as reflected by the increased activity of SOD, GSH-px and CAT following PBA intervention compared with that in the high-fructose group. These results suggest that ERS is connected to oxidative stress in fatty liver in high-fructose-fed rats. Previous studies have suggested that there is a complicated interaction between endoplasmic reticulum and oxidative stress. Specifically, free radicals, including reactive oxygen species (24), are one of the key messengers between the two cell events (25). Evidence suggests that endoplasmic reticulum is a potent source of ROS production. ROS produced in endoplasmic reticulum under ERS has been estimated to account for $\sim 25 \%$ of all ROS generated in cells. Sustained ERS can result in the accumulation of reactive oxygen species and promotes oxidative stress $(26,27)$. In addition, C/EBP homologous protein (CHOP) activation seems to enhance oxidative stress: as previously shown, CHOP dele- 
A

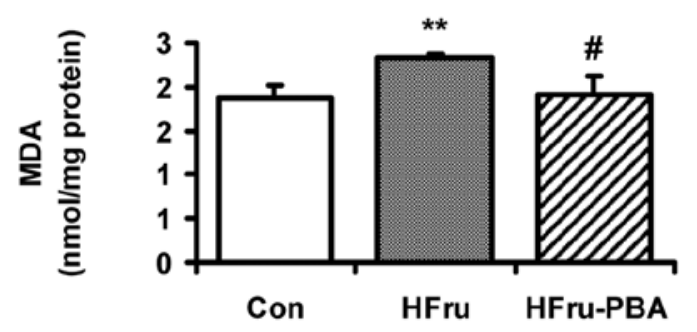

C

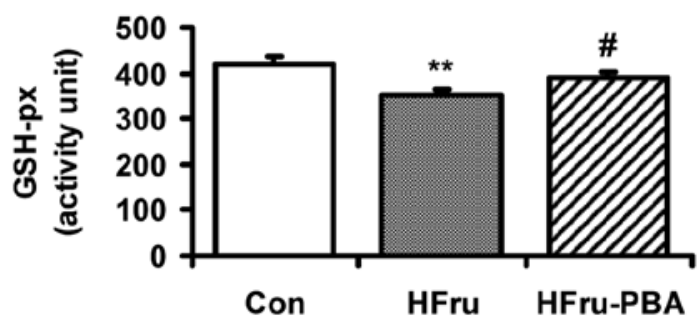

B

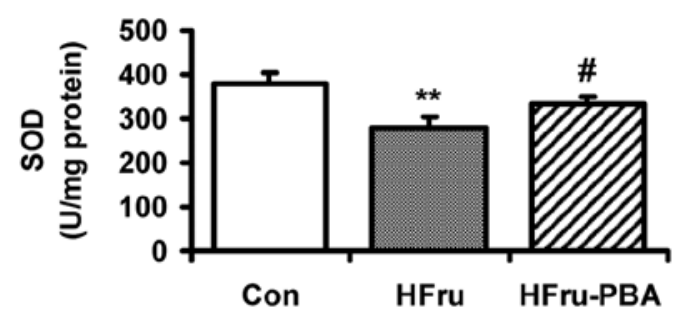

D

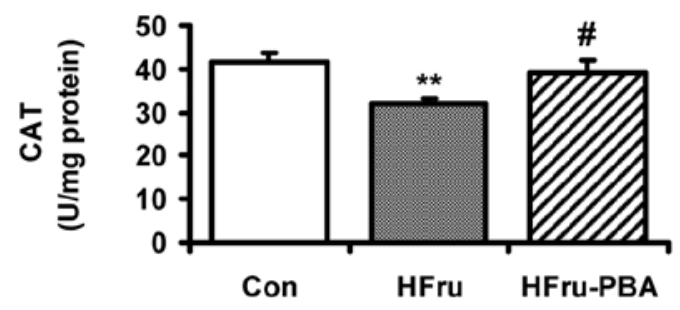

Figure 6. Oxidative stress in liver tissue in high-fructose-fed rats was relieved by PBA intervention. (A) MDA levels. The enzyme activities of (B) SOD, (C) GSH-px and (D) CAT in livers in three groups of rats. Data are presented as means $\pm \mathrm{SE} .{ }^{* *} \mathrm{P}<0.01$ vs. Con rats; ${ }^{\#} \mathrm{P}<0.05$ vs. HFru rats ( $\mathrm{n}=6$ ).

A

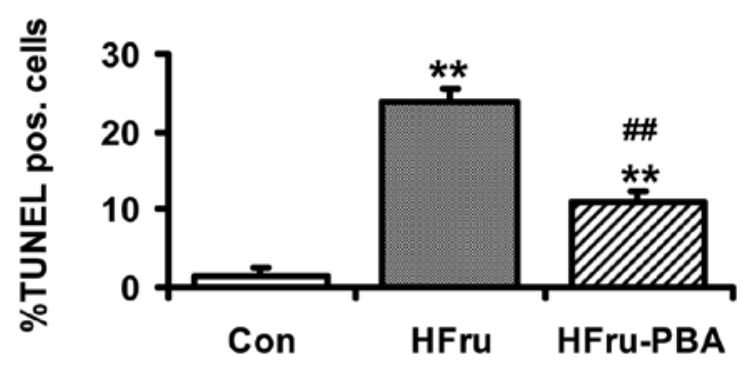

B

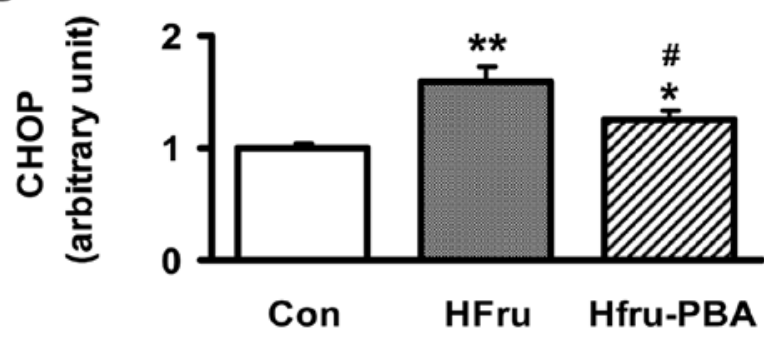

CHOP

$\beta$-actin

Figure 7. TUNEL assay and the protein expression of CHOP in three groups of rats after 8 weeks of feeding. (A) TUNEL assay of livers in the three groups of rats. (B) Protein levels of CHOP in the three groups of rats. Data are presented as means $\pm \mathrm{SE}$, and two representative blots of each group were provided for $\mathrm{CHOP}$ protein. ${ }^{*} \mathrm{P}<0.05$ vs. Con rats; ${ }^{* *} \mathrm{P}<0.01$ vs. Con rats; ${ }^{\#} \mathrm{P}<0.05$ vs. HFru rats; ${ }^{\# \#} \mathrm{P}<0.01$ vs. HFru rats $(\mathrm{n}=6)$. tion reduces oxidative damage in mouse models of diabetes (28). By contrast, UPR in ERS has a protective effect against oxidative stress response via the PERK and IRE-1-XBP1 pathways (29). The PERK pathway provides protection from ROS via eIF $2 \alpha$. Mice lacking the ability to phosphorylate eIF $2 \alpha$ are characterized by a severe diabetic phenotype that can be attenuated by a high antioxidant diet, suggesting a role for eIF $2 \alpha$ phosphorylation in the prevention of oxidative stress (30). XBP1 may also protect cells from oxidative damage. Mouse embryonic fibroblasts deficient in XBP1 were more prone to cell death and less able to activate antioxidant defenses following exposure to hydrogen peroxide (31). The results in the present study support that ERS and oxidative stress are cross-linked in the development of fatty liver and that inhibition of ERS can affect oxidative stress. However, the interaction and the cause-effect relationship between the two events remain unclear. Further investigations are needed to clarify the relationship between the two stress responses in the development of hepatic steatosis.

Besides relieving oxidative stress, apoptosis in liver cells has been significantly decreased by PBA inhibition in highfructose-fed rats. Apoptosis is a cell event occurring in the development of NAFLD and is associated with ERS and oxidative stress (32-34). CHOP is best known as an important mediator of ERS-induced cell death. Chronic ERS promotes apoptosis, at least in part through the activation of CHOP (32). In addition to ERS, oxidative stress is a key mechanism responsible for liver cell death and liver damage (34). The accumulation of ROS (24) also contributes to the apoptosis of hepatocytes (35). Therefore, the decreased apoptotic rate and decreased expression of CHOP in liver in the HFru-PBA group may be the results of the improvement of ERS and oxidative stress following PBA intervention. 
Findings of this study have proven that long-term highfructose intake is capable of inducing ERS in liver with the occurrence of hepatic steatosis. PBA intervention is able to improve the lipid accumulation induced by high-fructose feeding through inhibition of the upstream lipogenic transcriptional factors SREBP1c and XBP1 and downregulation of the lipogenic key enzymes. Besides ameliorating steatosis, oxidative stress and apoptosis in liver were also alleviated when ERS was resolved by PBA inhibition. The present study sheds new light on the role of ERS in the development of fatty liver. ERS is therefore a potential target for the prevention and treatment of NAFLD.

\section{Acknowledgements}

The authors thank Professor Jiming Ye (Health Innovations Research Institute, RMIT University, Melbourne, Victoria, Australia) for his advice on and interest in the study. This study has been supported by fundings from the Chinese National Science Foundation Grant (no. 81200639; allocation to L.-P.R.; 2012) and from the International Cooperation Foundation Grant in Science and Technology Department of Hebei Province (no. 11396406-D; allocation to G.-Y.S.; 2011).

\section{References}

1. Malavolti M, Battistini NC, Miglioli L, et al: Influence of lifestyle habits, nutritional status and insulin resistance in NAFLD. Front Biosci (Elite Ed) 4: 1015-1023, 2012.

2. Cankurtaran M, Tayfur O, Yavuz BB, Geyik S, Akhan O and Arslan S: Insulin resistance and metabolic syndrome in patients with NAFLD but without diabetes: effect of a 6 month regime intervention. Acta Gastroenterol Belg 70: 253-259, 2007.

3. Moore JB: Non-alcoholic fatty liver disease: the hepatic consequence of obesity and the metabolic syndrome. Proc Nutr Soc 69: 211-220, 2010.

4. Moore JB: Symposium 1: Overnutrition: consequences and solutions Non-alcoholic fatty liver disease: the hepatic consequence of obesity and the metabolic syndrome. Proc Nutr Soc $1-10,2010$.

5. Ouyang X, Cirillo P, Sautin Y, et al: Fructose consumption as a risk factor for non-alcoholic fatty liver disease. J Hepatol 48: 993-999, 2008.

6. Nomura K and Yamanouchi T: The role of fructose-enriched diets in mechanisms of nonalcoholic fatty liver disease. J Nutr Biochem 23: 203-208, 2012.

7. Yilmaz Y: Review article: fructose in non-alcoholic fatty liver disease. Aliment Pharmacol Ther 35: 1135-1144, 2012.

8. Kanuri G, Spruss A, Wagnerberger S, Bischoff SC and Bergheim I: Role of tumor necrosis factor $\alpha(\mathrm{TNF} \alpha)$ in the onset of fructose-induced nonalcoholic fatty liver disease in mice. J Nutr Biochem 22: 527-534, 2011.

9. Hsieh PS: Inflammatory change of fatty liver induced by intraportal low-dose lipopolysaccharide infusion deteriorates pancreatic insulin secretion in fructose-induced insulin-resistant rats. Liver Int 28: 1167-1175, 2008.

10. Aragno M, Tomasinelli CE, Vercellinatto I, et al: SREBP-1c in nonalcoholic fatty liver disease induced by Western-type high-fat diet plus fructose in rats. Free Radic Biol Med 47: 1067-1074, 2009.

11. Oyadomari S, Harding HP, Zhang Y, Oyadomari M and Ron D: Dephosphorylation of translation initiation factor 2alpha enhances glucose tolerance and attenuates hepatosteatosis in mice. Cell Metab 7: 520-532, 2008.

12. Kammoun HL, Chabanon H, Hainault I, et al: GRP78 expression inhibits insulin and ER stress-induced SREBP-1c activation and reduces hepatic steatosis in mice. J Clin Invest 119: 1201-1215, 2009.

13. Lee AH, Scapa EF, Cohen DE and Glimcher LH: Regulation of hepatic lipogenesis by the transcription factor XBP1. Science 320: 1492-1496, 2008.
14. Ozcan U, Cao Q, Yilmaz E, et al: Endoplasmic reticulum stress links obesity, insulin action, and type 2 diabetes. Science 306 : 457-461, 2004.

15. Ozcan U, Yilmaz E, Ozcan L, et al: Chemical chaperones reduce ER stress and restore glucose homeostasis in a mouse model of type 2 diabetes. Science 313: 1137-1140, 2006.

16. Yang JS, Kim JT, Jeon J, et al: Changes in hepatic gene expression upon oral administration of taurine-conjugated ursodeoxycholic acid in ob/ob mice. PLoS One 5: e13858, 2010.

17. Ren LP, Chan SM, Zeng XY, et al: Differing endoplasmic reticulum stress response to excess lipogenesis versus lipid oversupply in relation to hepatic steatosis and insulin resistance. PLoS One 7: e30816, 2012.

18. Sewter C, Berger D, Considine RV, et al: Human obesity and type 2 diabetes are associated with alterations in SREBP1 isoform expression that are reproduced ex vivo by tumor necrosis factor-alpha. Diabetes 51: 1035-1041, 2002.

19. Ye JM, Doyle PJ, Iglesias MA, Watson DG, Cooney GJ and Kraegen EW: Peroxisome proliferator-activated receptor (PPAR)-alpha activation lowers muscle lipids and improves insulin sensitivity in high fat-fed rats: comparison with PPAR-gamma activation. Diabetes 50: 411-417, 2001.

20. Boncompagni E, Gini E, Ferrigno A, et al: Decreased apoptosis in fatty livers submitted to subnormothermic machine-perfusion respect to cold storage. Eur J Histochem 55: e40, 2011.

21. Yu C, Chen Y, Cline GW, et al: Mechanism by which fatty acids inhibit insulin activation of insulin receptor substrate-1 (IRS-1)-associated phosphatidylinositol 3-kinase activity in muscle. J Biol Chem 277: 50230-50236, 2002.

22. Samuel VT: Fructose induced lipogenesis: from sugar to fat to insulin resistance. Trends Endocrinol Metab 22: 60-65, 2011.

23. Iizuka K, Bruick RK, Liang G, Horton JD and Uyeda K: Deficiency of carbohydrate response element-binding protein (ChREBP) reduces lipogenesis as well as glycolysis. Proc Natl Acad Sci USA 101: 7281-7286, 2004.

24. Mosbah IB, Zaouali MA, Martel C, et al: IGL-1 solution reduces endoplasmic reticulum stress and apoptosis in rat liver transplantation. Cell Death Dis 3: e279, 2012.

25. Malhotra JD and Kaufman RJ: Endoplasmic reticulum stress and oxidative stress: a vicious cycle or a double-edged sword? Antioxid Redox Signal 9: 2277-2293, 2007.

26. Shimizu Y and Hendershot LM: Oxidative folding: cellular strategies for dealing with the resultant equimolar production of reactive oxygen species. Antioxid Redox Signal 11: 2317-2331, 2009.

27. Cullinan SB and Diehl JA: Coordination of ER and oxidative stress signaling: the PERK/Nrf2 signaling pathway. Int J Biochem Cell Biol 38: 317-332, 2006.

28. Song B, Scheuner D, Ron D, Pennathur S and Kaufman RJ: Chop deletion reduces oxidative stress, improves beta cell function, and promotes cell survival in multiple mouse models of diabetes. J Clin Invest 118: 3378-3389, 2008.

29. Gentile CL, Frye MA and Pagliassotti MJ: Fatty acids and the endoplasmic reticulum in nonalcoholic fatty liver disease. Biofactors 37: 8-16, 2011.

30. Back SH, Scheuner D, Han J, et al: Translation attenuation through eIF2alpha phosphorylation prevents oxidative stress and maintains the differentiated state in beta cells. Cell Metab 10: 13-26, 2009.

31. Liu Y, Adachi M, Zhao S, et al: Preventing oxidative stress: a new role for XBP1. Cell Death Differ 16: 847-857, 2009.

32. Cao J, Dai DL, Yao L, et al: Saturated fatty acid induction of endoplasmic reticulum stress and apoptosis in human liver cells via the PERK/ATF4/CHOP signaling pathway. Mol Cell Biochem 364: 115-129, 2012.

33. Alkhouri N, Carter-Kent C and Feldstein AE: Apoptosis in nonalcoholic fatty liver disease: diagnostic and therapeutic implications. Expert Rev Gastroenterol Hepatol 5: 201-212, 2011.

34. Jin WP, Quan XQ, Meng FP, Cui XD and Piao HJ: Relationship among hepatocyte apoptosis, P450 2E1 and oxidative stress in alcoholic liver disease of rats. Zhongguo Wei Zhong Bing Ji Jiu Yi Xue 19: 419-421, 2007 (In Chinese).

35. Haynes CM, Titus EA and Cooper AA: Degradation of misfolded proteins prevents ER-derived oxidative stress and cell death. Mol Cell 15: 767-776, 2004. 